\title{
Autophagy and cardiometabolic risk factors
}

\author{
Juan G. Juárez-Rojas • Gissette Reyes-Soffer • \\ Donna Conlon • Henry N. Ginsberg
}

Published online: 23 September 2014

(C) The Author(s) 2014. This article is published with open access at Springerlink.com

\begin{abstract}
Autophagy is an essential cellular pathway by which protein aggregates, long-lived proteins, or defective organelles are sequestered in double membrane vesicles and then degraded upon fusion of those vesicles with lysosomes. Although autophagy plays a critical role in maintaining intracellular homeostasis and keeping the cell in a healthy state, this key pathway can become dysregulated in various cardiometabolic disorders, such as; obesity, dyslipidemia, inflammation, and insulin resistance. In these conditions, autophagy may actually worsen the pathological state instead of protecting the cell or organism. In this review, we discuss how dysregulated autophagy may be linked to increases in cardiovascular risk factors, and how manipulation of the autophagic machinery might reduce those risks.
\end{abstract}

Keywords Autophagy · Obesity · Dyslipidemia .

Inflammation · Insulin resistance, and cardiovascular disease

J. G. Juárez-Rojas · G. Reyes-Soffer • D. Conlon · H. N. Ginsberg Department of Medicine, Columbia University College of Physicians and Surgeons, New York, NY, USA

J. G. Juárez-Rojas

e-mail: gaboyk2@gmail.com

G. Reyes-Soffer

e-mail: gr2104@cumc.columbia.edu

D. Conlon

e-mail: dc2054@cumc.columbia.edu

J. G. Juárez-Rojas

Endocrinolgy Department, National Institute of Cardiology "Ignacio

Chávez", Mexico City, Mexico

H. N. Ginsberg $(\bowtie)$

PH10-305, Irving Institute for Clinical and Translational Research, 630 West 168 Street, New York, NY 10032, USA

e-mail: hng1@cumc.columbia.edu

\section{Introduction}

A detailed understanding of how cells maintain homeostasis through the balance of the synthesis and breakdown of proteins is critical to the development of new strategies to prevent and/or treat human disease states. Autophagy, or self-eating, is a general term for the catabolic processes, conserved from yeast to humans, that involves lysosomal degradation of cell constituents (including mitochondria, peroxisomes, and endoplasmic reticulum), unfolded or misfolded proteins, and intracellular pathogens. Autophagy can be induced by a change in cellular environment or in response to starvation when a rapid recycling of fatty acids and amino acids is required to ensure cell survival [1]. Association studies have found a link between autophagy and cardiovascular disease [2-4]; and recently, several publications have reported a strong relationship between autophagy and cardiometabolic risk factors such as obesity [5-7], dyslipidemia [8-10], inflammation [11-13], insulin resistance, and diabetes mellitus [14-16]. The objective of the present review is to describe how dysregulation of autophagy in these states can increase cardiovascular risk, and how manipulation of the autophagic machinery may reverse the disordered metabolism driving these risk factors.

\section{Autophagy}

When first described in 1966, autophagy was considered a nonselective process; however, the findings that different cytosolic proteins are degraded by lysosomes at different rates, and that some organelles or proteins are preferentially degraded under particular conditions, indicate a level of selectivity in lysosomedependent degradation [17]. Although constitutive mammalian autophagy is essential for basal cellular metabolism and homeostasis, increased levels of lysosomal degradation of cell components have been found under conditions of nutrient or 
oxygen deprivation, endoplasmic reticulum stress, proteasome malfunction, or damage caused by environment changes $[2$, $18]$.

There are three types of autophagy in the cell: macroautophagy (MAC-A), chaperone-mediated autophagy (CMA), and microautophagy (MIC-A). MAC-A is a process in which components of the cytoplasm, including long-lived proteins and organelles, are sequestered inside double-membrane vesicles called autophagosomes. The autophagosomes then fuse with lysosomes to form autophagolysosomes, where the contents are degraded and their molecular components released for recycling or energy production $[18,19]$. CMA is driven by the heat shock cognate (HSC) 70, which binds specific polypeptides or proteins containing a KFERQ motif and transports them directly into the lysosome, where interaction with the lysosome-associated membrane protein (LAMP) type 2A receptors leads to protein internalization and degradation $[20,21]$. Finally, in MIC-A, defective molecules or organelles are directly engulfed into the lysosomes for degradation and recycling of their components [22]. In this review we will focus on MAC-A.

The MAC-A process is divided in four different mechanistic steps: initiation, nucleation, autophagosome formation, and fusion of the autophagosome with the lysosome resulting in catabolism of the contents (Fig. 1). These processes are mediated by different sets of proteins, encoded by approximately 30 autophagy-related genes (ATGs) characterized in yeast, but highly conserved in eukaryotes cells $[4,18]$.

\subsection{Initiation}

The exact source of the lipids and/or membranes for the initial phagophore is currently unknown and being debated. Some researchers have reported that during initiation, recruitment of lipids from several organelles, depending on the cell type and stimulus, results in the formation of isolation membranes [18]. The initiation step is controlled by a complex of the uncoordinated 51-like kinase 1 (ULK1, also called Atg1), Atg13, and focal adhesion kinase interacting protein of $200 \mathrm{kD}$ (also called Atg17). It is suggested that mammalian Atg13 and Atg 17 are phosphorylated by ULK1 [23], and that ULK1 also undergoes autophosphorylation, which is conducive to a conformational change and autophagy induction [24]. Some studies have suggested that $A \operatorname{tg} 13$ is phosphorylated by either the mammalian target of rapamycin (mTOR) or ULKs on different residues, which may exert opposite effects on autophagy induction dependent on nutrient status [4]. The mammalian target of rapamycin complex 1 (mTORC1) is an important component of a network that senses the nutrient state of the cell and accordingly controls the levels of anabolism and catabolism to maintain homeostasis [25]. Low glucose levels or high levels of adenosine 5'-monophosphate (AMP), which indicates low cellular energy status, nutrient deprivation, or stress, activate AMP-activated protein kinase, which in turn inhibits mTORC1 activity and induces its dissociation from the ULK1-Atg13-Atg17 complex. As a result, ULK1 is enzymatically active and phosphorylates Atg13 and Atg17 to stimulate the MAC-A process $[25,26]$. In contrast, under normal physiological conditions, insulin and insulin like growth factor 1 indirectly induce mTORC 1 activity and inhibit autophagy by stimulating class 1 phosphoinositol 3kinase (PI3K) production of phosphatidylinositol 3phosphate (PI3P), which induces the Akt kinase at the plasma membrane, and promotes mTORC1 activity [27, 28]. High levels of amino acids also increase mTORC1 activity, by

\section{INITIATION}

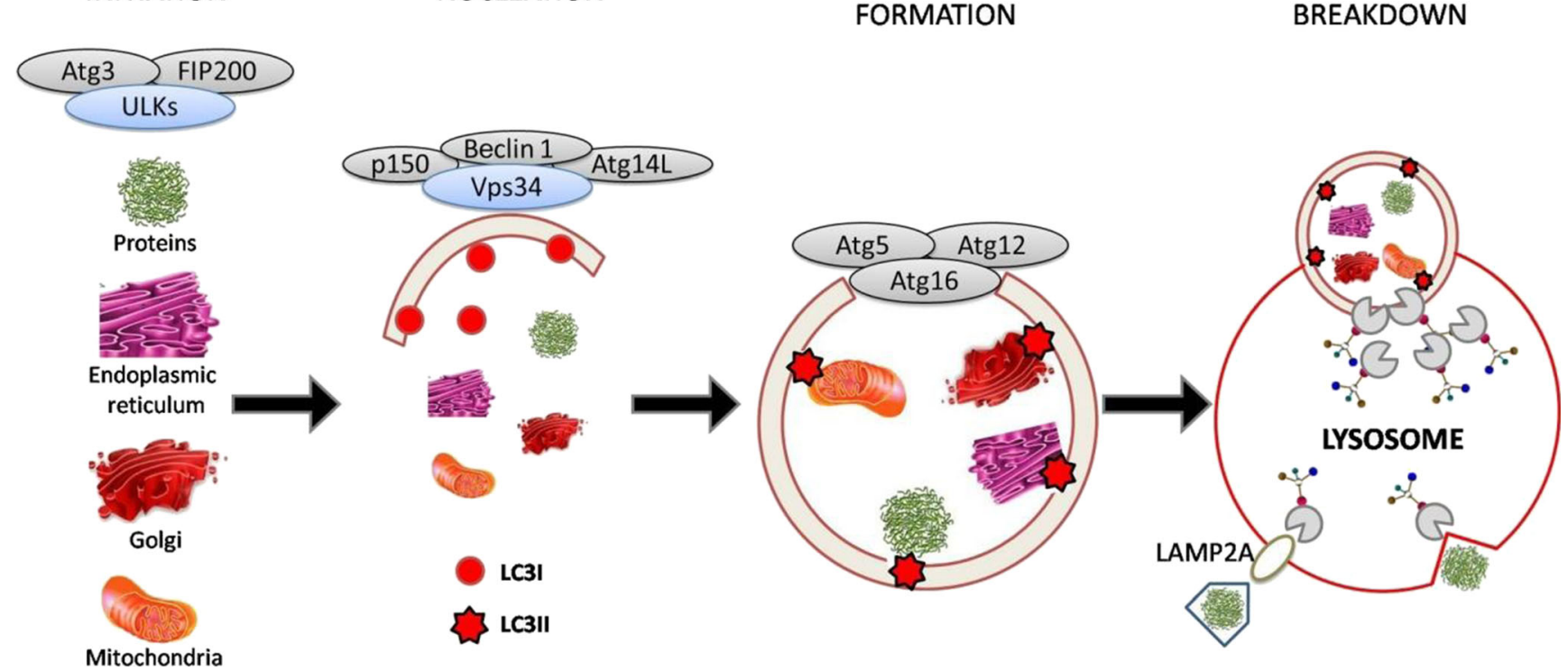

NUCLEATION

\section{AUTOPHAGOSOME FORMATION}

\section{FUSION AND} BREAKDOWN

Fig. 1 Schematic illustration of autophagy 
enhancing binding of this complex to regulatory proteins Rag and Rheb (Ras homolog enriched in brain) guanosine triphosphatases [29, 30].

\subsection{Nucleation}

This step requires a class III PI3K called vacuolar protein sorting 34 (Vps34) that forms a complex with Beclin 1 (also called Atg6/Vp330), p150 (also called Vps15) and Atg14L [31]. Production of PI3P by the Vps34 complex recruits WIPI (WD40 repeat protein interacting with phospho-inositides/ Atg18) to the isolation membrane, allowing recruitment of the light chain of the microtubule-associated protein 1 (LC3I/ Atg8) and further maturation of the autophagosome [32]. While specific inhibition of class I PI3K stimulates MAC-A indirectly through downstream Akt/mTOR inhibition, specific inhibition of Vps34 inhibits MAC-A through reduction of an autophagy-specific PI3P pool [33]. The function of Beclin 1 in autophagy has been shown to be regulated by B-cell lymphoma/leukemia-2, an anti-apoptotic protein that inhibits autophagy by binding and sequestering Beclin 1 under nutrient-rich conditions [4]. There are also other mTOR-independent autophagy pathways like c-Jun NH2-terminal kinase 1, which may induce autophagy by phosphorylating B-cell lymphoma/ leukemia-2 or Bim (a proapoptotic molecule which inhibits autophagy by its interaction with Beclin 1) and abolishing their inhibitory effects on autophagy [34].

\subsection{Autophagosome formation}

This step is mediated by two interdependent ubiquitin-like conjugation pathways. In one, Atg12 is activated by Atg7 (E1 activating enzyme) and attached to an internal lysine of the substrate protein Atg5 covalently. Atg12-Atg5 conjugation is constitutive and irreversible and further interacts with a coiled-coil protein Atg16, which allows the Atg12-Atg5Atg 16 complex to become a tetramer by selfoligomerization and also attaches it to the phagophore [4, 33]. The phagophore is an initial sequestering compartment, essential in regulating the membrane elongation and expansion that is essential for autophagosome formation. In the second pathway, LC3I is first cleaved by the Atg4 serine protease and then conjugated to phosphatidylethanolamine by $\operatorname{Atg} 7$ and $\operatorname{Atg} 3$ (E1 and E2 enzymes, respectively), allowing LC3I to engage the Atg12-Atg5-Atg16 complex on the phagophore. Once fixed to that complex, LC3I is lipidated to form LC3II. The two forms can be distinguished by Western blot analysis. After the autophagosome isolation membrane is formed, the Atg12-Atg5-Atg16 complex is released, whereas LC3II remains associated with the autophagosome until its fusion with the lysosome. In nutrient-rich conditions, the majority of LC3 is cytosolic; upon autophagy induction, LC3 largely exists as LC3II and is localized to both sides of the phagophore $[35,36]$.

\subsection{Vesicle fusion and autophagosome breakdown}

The autophagosomes-lysosomes fusion step is mediated by dynein-mediated transport of the autophagosomes along microtubules to fuse with endosomes or lysosomes. When autophagosome formation is completed, LC3II attached to the outer membrane is cleaved from phosphatidylethanolamine by Atg4 and released back to the cytosol [37]. In mammalian cells, the fusion event requires the LAMP type 2A receptor and the small GTPase, Rab7 [38, 39]; however, the exact mechanism remains to be demonstrated. After autophagosome and lysosome fusion, degradation of the inner vesicle is dependent on a series of lysosomal/vacuolar acid hydrolases, such as proteinases A and B (encoded by PEP4 and $P R B 1$, respectively) and cathepsins B, D and L [40]. The resulting small molecules generated from this degradation, particularly amino acids, are released back to the cytosol for re-utilization in protein synthesis and maintenance of cellular functions. The tricarboxylic acid cycle, which utilizes amino acids for generation of bioenergetic molecules and biosynthetic intermediates, appears to co-ordinate with MAC-A through negative feedback of pyruvate on MAC-A [4]. Lipid breakdown supplies free fatty acids needed to sustain cellular rates of mitochondrial $\beta$-oxidation and adenosine-5'-triphosphate generation, maintain cellular homeostasis, and promote the metabolism of potentially toxic lipid molecules [10].

\section{Obesity and autophagy}

Increasing rates of overweight and obesity worldwide are closely linked to incidence of metabolic disorders worldwide. Both conditions are associated with accumulation of adipose tissue. White adipose tissue (WAT) functions to store energy in the fed state by taking up free fatty acids and synthesizing $\mathrm{TG}$, which can later be hydrolyzed to provide fatty acids to other tissues during fasting or exercise. In contrast, brown adipose tissue (BAT) has a reduced capacity for lipid storage, but a high rate of $\beta$-oxidation that is mediated by the mitochondrial uncoupling protein $[10,41]$. Previous reports in cellular and animal models have shown that general obesity and metabolic disease correlate with decreased BAT activity, whereas resistance to metabolic disease from obesity correlates with increased BAT function or the induction of brown adipocyte-like gene expression in WAT [9, 10].

Autophagy has been implicated in cellular development and differentiation including adipogenesis. In a recent study, by Singh and colleagues [5], inhibition of autophagy with 3methyladenine or knockdown of Atg7 in 3T3-L1 pre- 
adipocytes reduced accumulation of TG and expression of transcription factors such as peroxisome proliferator-activated receptor gamma and CCAAT-enhancer-binding protein, which are involved in adipocyte differentiation. Inhibition of autophagy was also associated with decreased markers of differentiated adipocytes, such as fatty acid synthase, stearoyl-coenzyme A desaturase 1, and glucose transporter 4. Consistent with these findings, studies in Atg5-null murine embryonic fibroblasts showed a decrease in adipocyte differentiation and lipid accumulation [42]. Additionally, mice with targeted knockout of Atg7 in adipose tissue have decreased WAT mass and increased mitochondrial uncoupling protein and mitochondrial enzymes, leading to a $\sim 32$ fold higher $\beta$-oxidation in adipose tissue, which suggests that the decrease in lipid storage was secondary to a failure in differentiation and not a direct effect on lipid metabolism [5]. The transformation of WAT from an energystoring tissue into an energy-expending tissue resulted in a leaner mouse that was resistant to high fat diet-induced obesity, had lower plasma concentrations of free fatty acids, triglycerides and cholesterol, and showed a markedly increased sensitivity to insulin $[5,10]$. In line with these results, human studies revealed that autophagy is upregulated in obese individuals, who have increased expression of autophagy-related proteins Atg5, LC3I and LC3II, as well as elevated autophagic flux in both omental and subcutaneous adipose tissue $[6,7]$. These results suggest that blocking autophagy in adipose tissue might lead to reduced WAT and increased BAT or beige/browned WAT with greater $\beta$-oxidation. Such a change in the balance of WAT and BAT would decrease obesity and some of the associated metabolic disorders [43].

\section{Lipoprotein metabolism and autophagy}

Abnormalities in lipoprotein metabolism leading to dyslipidemias are important in atherogenesis and the development of clinical cardiovascular disease. Lipoproteins are macromolecular spherical complexes of lipids and proteins that carry hydrophobic neutral lipids such as cholesterol esters and triglycerides in a core surrounded by amphipathic phospholipids, free cholesterol, and apolipoproteins. The structure of the lipoprotein allows the hydrophobic core lipids to traverse the hydrophilic environment of plasma. Lipoproteins can be classified according to their hydrated density into chylomicrons, very-low-density-lipoproteins (VLDL), intermediate-density lipoproteins (IDL), low-density lipoproteins (LDL) and high density lipoproteins (HDL) [44, 45]. The role of VLDL (synthesized in liver) and chylomicrons (synthesized in intestine) is to deliver energy, in the form of triglycerides, to peripheral tissues using lipoprotein lipase to generate free fatty acids. After release of fatty acids, the remnants of VLDL and chylomicrons return to the liver where they are taken up to deliver their remaining triglycerides and most of their cholesterol; varying proportions of VLDL remnants and nearly all chylomicron remnants are removed by the liver. Remnant lipoprotein cholesterol plays a key role in the regulation of hepatic cholesterol homeostasis, including regulation of cholesterol synthesis and LDL receptor expression. The VLDL that is not removed by the liver is converted to LDL after passing through the IDL state. VLDL, chylomicron remnants, IDL and LDL, which all carry apolipoprotein $\mathrm{B}$, are each atherogenic because they can permeate the endothelial layer of arteries and initiate and/or increase atherosclerotic plaque formation $[10,45]$.

Apolipoprotein B100 (apoB100) is a protein synthesized in liver that is essential to the formation of VLDL, IDL and LDL. Considering the critical role that apoB100 plays in lipid transport, metabolic regulation, and atherogenesis, it is not surprising that regulation of apoB100 itself, or apoB100-containing lipoproteins, is complex and occurs at several stages [45]. Multiple apoB100 degradative pathways have been described, and dysregulation of some of these pathways may contribute to the overproduction of VLDL concomitant with increased plasma triglyceride and LDL levels in blood. Factors associated with such dysregulation of hepatic apoB100 metabolism include insulin resistant states such as obesity, metabolic syndrome, and type 2 diabetes mellitus [46, 47].

Like all secreted proteins, apoB100 is synthesized at the surface of the endoplasmic reticulum (ER), where the protein is lipidated during its translocation across the ER membrane by microsomal triglyceride transfer protein (MTP) (Fig. 2). This lipidation allows apoB100 to complete translocation and initiate the assembly of VLDL. In lipid-poor conditions or in absence of MTP, a large proportion of newly synthesized apoB100 is cotranslationally ubiquitinylated and degraded by the proteasome. However, some experimental models have shown that approximately $50 \%$ or more apoB100 degradation occurs after translocation, and this is mainly non-proteosomal degradation [45, 48]. The co-localization of proteosomes, autophagosomes, and apoB100 in structures resembling LDs has suggested the involvement of autophagic mechanisms in apoB100 degradation [49]. Furthermore, when the concentration of misfolded proteins rises in the ER, the unfolded protein response is triggered, leading to increased synthesis of ER chaperones and activation of other degradation pathways, including autophagy [45, 50]. Other studies have shown that increased delivery of omega-3 poly-unsaturated fatty acids induce autophagicapoB100 degradation, reducing the secretion of VLDL [51, 52]. The induction of this degradation most likely occurs in the Golgi (post-ER presecretory proteolysis), where the VLDL-apoB100 undergoes aggregation after exposure to lipid peroxides. In contrast to proteosomal degradation, post-ER presecretory proteolysis can occur even when lipid availability and MTP are normal. These data suggest a potential therapeutic role for autophagy in states of increased VLDL secretion and hyperlipidemia. Previous studies have shown that HDL are lipoproteins with antioxidant, anti-apoptotic, and anti-inflammatory properties. A recent study 
(a) Normal assembly and secretion of apoB100/VLDL

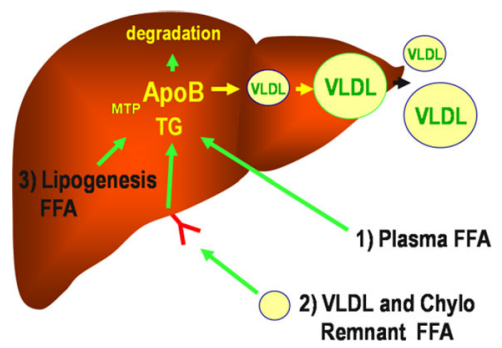

(c) Severe ER stress with steatosis

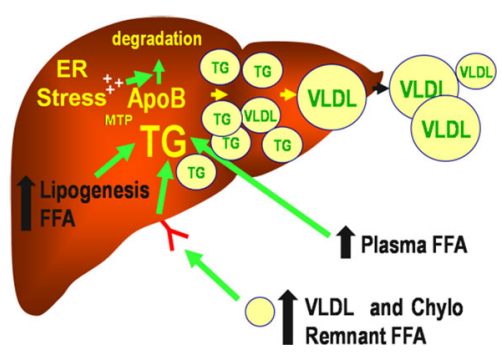

Fig. 2 Forces regulating the assembly and secretion of apoB100/VLDL. ApoB100/VLDL is a vehicle for transporting energy from the liver to the periphery and its assembly and secretion are extremely complex. a Nascent lipid-poor apoB100 is either targeted for complete translocation into the lumen of the endoplasmic reticulum (ER) for assembly into VLDL or for co- and post-translational ER degradation. The fate of nascent apoB is dependent on lipidation with TG via the action of microsomal triglyceride transfer protein (MTP). The TG originates from free fatty acids (FFA) derived from three sources: 1) lipolysis of adipose tissue, 2) VLDL and chylomicron remnants returning to the liver, and 3) hepatic de novo lipogenesis. The VLDL formed in this step then matures with addition of more core lipids, principally TG. This second step is also regulated by MTP and occurs either in the ER or in a post-ER

indicated that HDL may also prevent the expression of LC3II and Beclin 1 in human endothelial cells, thereby preventing the autophagy triggered by oxidized LDL [53].

The fact than during fed state, both autophagy and lipolysis are suppressed (leading to cellular lipid storage for future energy demands), and that during periods of limited nutrient supply autophagy increases in parallel with lipolysis, suggest a potential interrelationship between these two pathways. In this respect, very interesting in vitro and in vivo experiments indicate that inhibition of MAC-A by 3-methyladenine or knockdown of Atg5 or Atg7 genes significantly increased hepatic triglycerides and cholesterol content in mice during prolonged fasting, when delivery of fatty acids to the liver was dramatically increased [7]. The increased hepatic lipid content was present in lipid droplets (LDs), which are cytosolic structures where lipids are stored as a central core of triglycerides and cholesterol esters surrounded by a phospholipid monolayer and associated proteins [54]. Prolonged fasting was also associated with: 1) decreased rates of lipolysis and fatty acid $\beta$-oxidation; 2) movement of lipids through the autophagic pathway, indicated by colocalization of neutral lipids with markers of autophagic vacuoles and lysosomes, and by findings of LDs

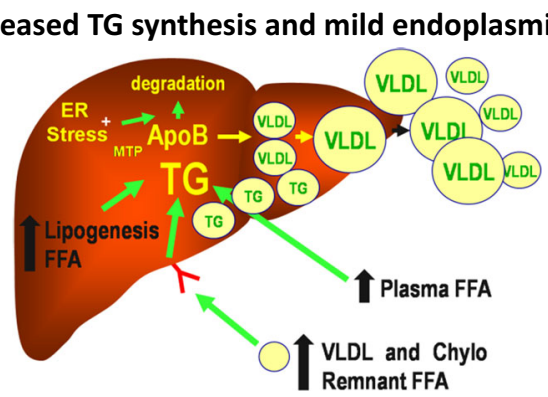

Remnant FFA

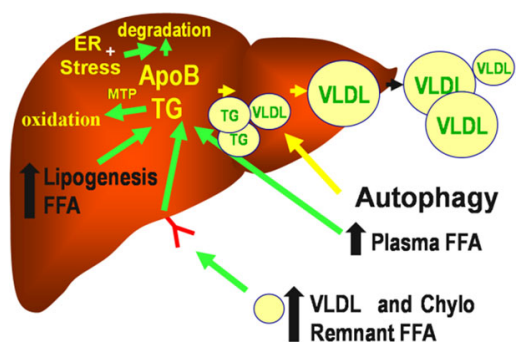

compartment, such as the Golgi. At this point, mature VLDL can be secreted. b When TG synthesis from any source is increased, the response is an increase in VLDL secretion. However, mild or moderate ER stress can also result. ER stress can cause increased degradation of apoB leading to less than maximal VLDL secretion and some steatosis. $\mathbf{c}$ If ER stress becomes even more severe, degradation of apoB increases further and VLDL secretion can fall even more, leading to significant steatosis. d Under certain conditions, autophagy can be induced at this point and VLDL can be diverted for post-ER/Golgi degradation. Lipophagy and ER autophagy may also occur, resulting in increased fatty acid oxidation and less steatosis. Thus autophagy might promote both less hepatic steatosis and less apoB100/VLDL secretion

within autophagic vacuoles; and 3) direct interaction of LC3 with LDs before autophagosome formation [7]. These results indicate that autophagy may play a role in the regulation of hepatocyte lipid content, through breakdown of LDs stored triglycerides and cholesterol, by a pathway the authors called lipophagy $[9,10]$. This alternative pathway for lipid metabolism may provide hepatocytes with the ability to mobilize large amount of bulk lipids for oxidation when other sources of energy are very limited. These results also suggest that activation of autophagy/lipophagy might be an approach to reducing hepatic steatosis.

\section{Inflammation, atherosclerosis and autophagy}

Inflammation is the first response of the immune system to infection or tissue injury. Although the initial inflammatory response is beneficial, prolonged or chronic inflammation is detrimental, playing a role in diseases such as atherosclerosis [55]. Atherosclerotic lesions begin with dysregulated lipid metabolism resulting in lipoproteins infiltrating the vessel wall, followed by macrophages, leading to the development 
of lipid-laden foam cells filled with cholesterol ester (CE)enrich LDs $[10,56]$. Breakdown of foam cell CEs by hydrolases is pivotal in the cholesterol mobilization from these cells. In this respect, it has been shown recently that lipid loading activates autophagy in macrophages, thereby targeting LDs for delivery to lysosomes for lipolysis. This allows cholesterol mobilization from LDs for adenosine-5'-triphosphate binding cassette transporter-mediated cholesterol efflux and macrophage reverse cholesterol transport [11]. Importantly, inhibition of macrophage lysosomes only affects cholesterol efflux from lipid-loaded, but not normal cells, indicating a specific role for lysosomal degradation in foam cells [11]. The authors of this study also observed that in lipid-loaded Atg5 knockout macrophages, cholesterol efflux was decreased, but that inhibition of lysosomal acid lipase had no additional effect on cholesterol efflux, suggesting that decreased autophagic trafficking of CEs to lysosomes was the cause of the decreased cholesterol efflux [11]. Two additional studies support these results and showed that activated macrophage autophagy prevents cellular CE accumulation [14], and promotes resistance to foam cell formation and atherosclerosis [13].

Some in vitro studies have also shown that autophagy can be stimulated in atherosclerotic lesions by oxidized lipids [3, 57], reactive oxygen species [58], endoplasmic reticulum stress [59], inflammation [60], and metabolic stress [61]. Although it is possible that basal stimulation of autophagy could be atheroprotective in macrophages, it is also possible that autophagy becomes dysfunctional in more advanced stages of atherosclerosis and loss of autophagic function promotes atherosclerosis, in part, through activation of the inflammasome [62]. Loss of autophagy had been associated with induction of the inflammasome and interleukin- $1 \beta$ processing in macrophages, possibly through either the loss of autophagic suppression of reactive oxygen species accumulation or reduced clearance of molecules that activate the inflammasome [63, 64]. Finally, defective macrophage autophagy has been shown to increases lesion and necrotic area in late atherosclerotic plaques, due to defective efferocytosis of apoptotic plaque macrophages [65]. Autophagy has an important role in the processing of efferocytosed material and has been highlighted as a key mechanism to induce nitric oxide production and reduce reactive oxygen species accumulations [66], which are both also closely related to atherogenesis.

\section{Insulin resistance/diabetes mellitus and autophagy}

Insulin is the major anabolic hormones produced by the pancreatic $\beta$ cells, and is essential for growth, development, and homeostasis of glucose, fat, and protein metabolism. Binding of insulin to its transmembrane receptor stimulates the intrinsic tyrosine kinase activity of the receptor, which phosphorylates target proteins, such as insulin receptor substrates 1 to 4, to trigger two major kinase cascades, the PI3K and the mitogen-activated protein kinase pathways, that mediate the metabolic and growth-promoting functions of insulin, respectively $[67,68]$. Individuals with insulin resistance have increased plasma insulin concentrations resulting mainly from increased insulin production, which is necessary to compensate for resistance to insulin's actions in hepatic, adipose, and muscle tissues. Insulin resistance is associated with excess body weight, physical inactivity, aging, inflammation, type 2 diabetes mellitus, and cardiovascular disease [69].

The fact that responsiveness to insulin is affected by several processes such as lipid accumulation, reactive oxygen species production, inflammation, ER stress, and mitochondrial turnover, suggest a relationship between insulin response and autophagy pathway. During nutrient deprivation, glucagon causes upregulation of autophagy, whereas excess nutrient supply leads to downregulation of autophagy through insulin signaling [14]. Furthermore, hyperinsulinemic, high fat dietfed mice [17], as well as obese (ob/ob) mice [18], display impaired hepatic autophagy, as indicated by low levels of LC3II and high levels of p62 (a protein normally degraded via autophagy). Of note, decreased autophagy in ob/ob mice was associated with decreased insulin signaling and increased ER stress, both of which were rescued by Atg7 overexpression [16]. Furthermore, studies in mice with selective deficiency of autophagy in $\beta$-cells demonstrated reduced conversion of LC3I to LC3II, marked accumulation of p62, and large protein aggregates in the cytosol of those cells. Importantly, reductions in basal autophagy levels allowed intracellular accumulation of damaged organelles, particularly dysfunctional mitochondria, that promoted increases in reactive oxygen species and reductions in glucose-stimulated insulin secretion in these mice $[70,71]$, who additionally developed diabetes with marked $\beta$-cell loss when fed a high-fat diet [72].

Considering that hyperinsulinemic states decreases autophagy, and that decreased autophagy can, in turn, downregulate insulin signaling, it is clear that autophagy and insulin signaling participate in a feedback mechanism with reciprocal regulation. The molecular basis for this reciprocity is via insulin signaling-mediated activation of mTORC1 during adequate substrate availability and mTORC1 inhibition of insulin signaling during decreased substrate availability [25, 28]. Although autophagy also may protect $\beta$-cell function [73], accelerated autophagy also appears to be involved in $\beta$-cell death under special conditions [67].

\section{Potential targets in the autophagic machinery}

Most of the results previously discussed suggest that regulation of autophagy could be a useful target to prevent or control some of the metabolic abnormalities associated with 
cardiovascular disease or atherosclerosis itself (Table 1). For example, experimental studies have shown that blocking pathways that stimulate autophagy, particularly in adipose tissue, may reduce WAT and increase $\beta$-oxidation; which in turn would decrease obesity and the associated metabolic disorders. It is important to highlight that any attempt to inhibit autophagy to prevent or treat obesity must first address important questions as to how such inhibition might affect mitophagy (process to remove damaged mitochondria through autophagy) in mature adipose tissue, as well as what would be the systemic effects of this inhibition [9, 74]. Elucidating the answers to these questions could provide the basis for novel approaches to converting WAT to BAT and effectively treating obesity and its associated metabolic disorders [43]. Conversely, activators of autophagy such as rapamycin and its analogs, everolimus and sirolimus, which act via mTOR inhibition, have shown promise, in preclinical studies, as beneficial potential therapeutics for coronary heart disease. These mTOR inhibitors, routinely used in drug eluting stents, were found to prevent atherosclerosis development and progression in atherogenic mouse models [75, 76], as well as specifically reduce the cholesterol content of the aortic arch [77]. Furthermore, a selective clearance of lesional macrophages and rise in apolipoprotein A1 and HDL were observed after rapamycin or sirolimus treatment, which suggests another plausible mechanism by which mTOR inhibition exerts atheroprotective effects [78, 79]. Although pharmacological

Table 1 Effects of modifying autophagy on cardiovascular risk factors

\begin{tabular}{|c|c|c|}
\hline $\begin{array}{l}\text { Cardiovascular } \\
\text { Risk factor }\end{array}$ & Potential target & Effect \\
\hline Obesity & $\begin{array}{l}\text { Decrease } \\
\text { autophagy }\end{array}$ & $\begin{array}{l}\downarrow \text { Triglycerides in plasma } \\
\downarrow \text { Cholesterol in plasma } \\
\downarrow \text { Lipid store } \\
\uparrow \text { Free fatty acid } \beta \text {-oxidation } \\
\uparrow \text { Obesity resistance }\end{array}$ \\
\hline Dyslipidemia & $\begin{array}{l}\text { Increase } \\
\text { autophagy }\end{array}$ & $\begin{array}{l}\downarrow \text { Triglycerides in plasma } \\
\downarrow \text { Cholesterol in plasma } \\
\downarrow \text { Lipid store } \\
\downarrow \text { Low density lipoprotein oxidation } \\
\uparrow \text { Free fatty acid } \beta \text {-oxidation } \\
\uparrow \text { Folding and traffic proteins }\end{array}$ \\
\hline Inflammation & $\begin{array}{l}\text { Increase } \\
\text { autophagy }\end{array}$ & $\begin{array}{l}\downarrow \text { Inflammasome } \\
\downarrow \text { Foam cell formation } \\
\downarrow \text { Cholesterol ester acummulation } \\
\downarrow \text { Reactive oxygen species } \\
\uparrow \text { Nitric oxide } \\
\uparrow \text { Transport reverse cholesterol } \\
\uparrow \text { Lipophagy }\end{array}$ \\
\hline $\begin{array}{l}\text { Insulin } \\
\quad \text { Resistance }\end{array}$ & $\begin{array}{l}\text { Increase } \\
\text { autophagy }\end{array}$ & $\begin{array}{l}\downarrow \text { Damaged organelles } \\
\downarrow \text { Mitochondrial dysfunction } \\
\downarrow \text { Reactive oxygen species } \\
\uparrow \text { Endoplasmic reticulum stress } \\
\uparrow \text { Glucose stimulated insulin secretion } \\
\uparrow \text { Insulin sensitivity }\end{array}$ \\
\hline
\end{tabular}

inhibition of mTOR can increase plasma levels of LDLcholesterol and triglycerides [80], as well as proinflammatory cytokines such as interleukin-6, monocyte chemoattractant protein-1, and tumor necrosis factor- $\alpha$ [81], combined treatment with lipid-lowering therapy or anti-inflammatory agents, may help to prevent the adverse effects of drug-induced autophagy without affecting the ability of these agents to deplete lipids and reduce macrophages with in atherosclerotic plaques $[80,81]$.

\section{Conclusions}

Autophagy is primarily a protective process that plays a role in recycling cellular constituents and maintaining cell homeostasis. Based on the literature reviewed here-in, it appears possible that reducing autophagy in adipose tissue might be an approach to controlling obesity whereas increasing autophagy in the liver, $\beta$ cell, or atherosclerotic lesion might provide protection from hyperlipidemia, diabetes mellitus, and coronary events secondary to rupture of necrotic atherosclerotic plaques. However, developing tissue specific approaches with narrow therapeutic benefit:risk ratios will be very challenging.

Acknowledgments Juarez-Rojas JG is grateful to Gobierno del Distrito Federal and ConsejoNacional de Ciencia y Tecnología (CONACyT; grant 186259), for the financial support for a postdoctoral fellowship at Columbia University. This work was also supported by grants from the National Institutes of Health: R01 HL55638 (H.G.); R01 HL110418 (H.G., J.R.-S.) and T32 DK007328 (D.C.).

Conflict of Interest The authors have no conflicts related to this work.

Open Access This article is distributed under the terms of the Creative Commons Attribution License which permits any use, distribution, and reproduction in any medium, provided the original author(s) and the source are credited.

\section{References}

1. Levine B, Klionsky DJ. Development by self-digestion: molecular mechanisms and biological functions of autophagy. Dev Cell. 2004;6(4):463-77.

2. Gustafsson AB, Gottlieb RA. Autophagy in ischemic heart disease. Circ Res. 2009;104(2):150-8.

3. Martinet W, De Meyer I, Verheye S, Schrijvers DM, Timmermans JP, De Meyer GR. Drug-induced macrophage autophagy in atherosclerosis: for better or worse? Basic Res Cardiol. 2013;108(1):321.

4. He C, Klionsky DJ. Regulation mechanisms and signaling pathways of autophagy. Annu Rev Genet. 2009;43:67-93.

5. Singh R, Xiang Y, Wang Y, Baikati K, Cuervo AM, Luu YK, et al. Autophagy regulates adipose mass and differentiation in mice. J Clin Invest. 2009;119(11):3329-39.

6. Kovsan J, Blüher M, Tarnovscki T, Klöting N, Kirshtein B, Madar L, et al. Altered autophagy in human adipose tissues in obesity. J Clin Endocrinol Metab. 2011;96(2):E268-77.

7. Ost A, Svensson K, Ruishalme I, Brännmark C, Franck N, Krook H, et al. Attenuated mTOR signaling and enhanced autophagy in 
adipocytes from obese patients with type 2 diabetes. Mol Med. 2010;16(7-8):235-46.

8. Singh R, Kaushik S, Wang Y, Xiang Y, Novak I, Komatsu M, et al. Autophagy regulates lipid metabolism. Nature. 2009;458(7242):1131-5.

9. Czaja MJ. Autophagy in health and disease. 2. Regulation of lipid metabolism and storage by autophagy: pathophysiological implications. Am J Physiol Cell Physiol. 2010;298(5):C973-8.

10. Liu K, Czaja MJ. Regulation of lipid stores and metabolism by lipophagy. Cell Death Differ. 2013;20(1):3-11.

11. Ouimet M, Franklin V, Mak E, Liao X, Tabas I, Marcel YL. Autophagy regulates cholesterol efflux from macrophage foam cells via lysosomal acid lipase. Cell Metab. 2011;13(6):655-67.

12. Mei S, Gu H, Ward A, Yang X, Guo H, He K, et al. p38 mitogenactivated protein kinase (MAPK) promotes cholesterol ester accumulation in macrophages through inhibition of macroautophagy. J Biol Chem. 2012;287(15):11761-8

13. Le Guezennec X, Brichkina A, Huang YF, Kostromina E, Han W, Bulavin DV. Wip1-dependent regulation of autophagy, obesity, and atherosclerosis. Cell Metab. 2012;16(1):68-80.

14. Finn PF, Dice JF. Proteolytic and lipolytic responses to starvation. Nutrition. 2006;22(7-8):830-44.

15. Yecies JL, Manning BD. Transcriptional control of cellular metabolism by mTOR signaling. Cancer Res. 2011;71(8):2815-20.

16. Yang L, Li P, Fu S, Calay ES, Hotamisligil GS. Defective hepatic autophagy in obesity promotes ER stress and causes insulin resistance. Cell Metab. 2010;11(6):467-78.

17. Kaushik S, Cuervo AM. Chaperones in autophagy. Pharmacol Res. 2012;66(6):484-93.

18. Benbrook DM, Long A. Integration of autophagy, proteasomal degradation, unfolded protein response and apoptosis. Exp Oncol. 2012;34(3):286-97.

19. Yorimitsu T, Klionsky DJ. Autophagy: molecular machinery for selfeating. Cell Death Differ. 2005;12 Suppl 2:1542-52.

20. Li W, Yang Q, Mao Z. Chaperone-mediated autophagy: machinery, regulation and biological consequences. Cell Mol Life Sci. 2011;68(5):749-63.

21. Arias E, Cuervo AM. Chaperone-mediated autophagy in protein quality control. Curr Opin Cell Biol. 2011;23(2):184-9.

22. Li WW, Li J, Bao JK. Microautophagy: lesser-known self-eating. Cell Mol Life Sci. 2012;69(7):1125-36.

23. Jung CH, Jun CB, Ro SH, Kim YM, Otto NM, Kundu M, et al. ULKAtg13-FIP200 complexes mediate mTOR signaling to the autophagy machinery. Mol Biol Cell. 2009;20(7):1992-2003.

24. Mizushima N, Komatsu M. Autophagy: renovation of cells and tissues. Cell. 2011;147(4):728-41.

25. Inoki K, Kim J, Guan K-L. AMPK and mTOR in cellular energy homeostasis and drug targets. Annu Rev Pharmacol Toxicol. 2012;52(2):381-400.

26. Petiot A, Ogier-Denis E, Blommaart EF, Meijer AJ, Codogno P. Distinct classes of phosphatidylinositol 3'-kinases are involved in signaling pathways that control macroautophagy in HT-29 cells. J Biol Chem. 2000;275(2):992-8.

27. Avruch J, Hara K, Lin Y, Liu M, Long X, Ortiz-Vega S, et al. Insulin and amino-acid regulation of mTOR signaling and kinase activity through the RhebGTPase. Oncogene. 2006;25(48):6361-72.

28. Benbrook DM, Masamha CP. The pro-survival function of Akt kinase can be overridden or altered to contribute to induction of apoptosis. Curr Cancer Drug Targets. 2011;11(5):586-99.

29. Kim E, Goraksha-Hicks P, Li L, Neufeld TP, Guan KL. Regulation of TORC1 by Rag GTPases in nutrient response. Nat Cell Biol. 2008;10(8):935-45.

30. Sancak Y, Peterson TR, Shaul YD, Lindquist RA, Thoreen CC, BarPeled L, et al. The Rag GTPases bind Raptor and mediate amino acid signaling to mTORC1. Science. 2008;320(5882):1496-501.

31. Lindmo K, Stenmark H. Regulation of membrane traffic by phosphoinositide 3-kinases. J Cell Sci. 2006;119(Pt 4):605-14.
32. Polson HE, de Lartigue J, Rigden DJ, Reedijk M, Urbé S, Clague MJ, et al. Mammalian Atg18 (WIPI2) localizes to omegasome-anchored phagophores and positively regulates LC3 lipidation. Autophagy. 2010;6(4):506-22.

33. Mizushima N, Noda T, Ohsumi Y. Apg16p is required for the function of the Apg12p-Apg5p conjugate in the yeast autophagy pathway. EMBO J. 1999;18(14):3888-96.

34. Lee MJ, Lee JH, Rubinsztein DC. Tau degradation: the ubiquitinproteasome system versus the autophagy-lysosome system. Prog Neurobiol. 2013;105(6):49-59.

35. Kabeya Y, Mizushima N, Ueno T, Yamamoto A, Kirisako T, Noda T, et al. LC3, a mammalian homologue of yeast Apg8p, is localized in autophagosome membranes after processing. EMBO J. 2000;19(21): $5720-8$.

36. Kirisako T, Baba M, Ishihara N, Miyazawa K, Ohsumi M, Yoshimori $\mathrm{T}$, et al. Formation process of autophagosome is traced with Apg8/Aut7p in yeast. J Cell Biol. 1999;147(2):435-46.

37. Kirisako T, Ichimura Y, Okada H, Kabeya Y, Mizushima N, Yoshimori T, et al. The reversible modification regulates the membrane-binding state of Apg8/Aut7 essential for autophagy and the cytoplasm to vacuole targeting pathway. J Cell Biol. 2000;151(2):263-76.

38. Jäger S, Bucci C, Tanida I, Ueno T, Kominami E, Saftig P, et al. Role for Rab7 in maturation of late autophagic vacuoles. J Cell Sci. 2004;117(Pt 20):4837-48.

39. Tanaka Y, Guhde G, Suter A, Eskelinen EL, Hartmann D, LüllmannRauch R, et al. Accumulation of autophagic vacuoles and cardiomyopathy in LAMP-2-deficient mice. Nature. 2000;406(6798):902-6.

40. Tanida I, Minematsu-Ikeguchi N, Ueno T, Kominami E. Lysosomal turnover, but not a cellular level, of endogenous LC3 is a marker for autophagy. Autophagy. 2005;1(2):84-91.

41. Lavallard VJ, Meijer AJ, Codogno P, Gual P. Autophagy, signaling and obesity. Pharmacol Res. 2012;66(6):513-25.

42. Baerga R, Zhang Y, Chen PH, Goldman S, Jin S. Targeted deletion of autophagy-related 5 (atg5) impairs adipogenesis in a cellular model and in mice. Autophagy. 2009;5(8):1118-30.

43. Qiang L, Wang L, Kon N, Zhao W, Lee S, Zhang Y, et al. Brown remodeling of white adipose tissue by SirT1-dependent deacetylation of Ppar $\gamma$. Cell. 2012;150(3):620-32.

44. Dominiczak MH, Caslake MJ. Apolipoproteins: metabolic role and clinical biochemistry applications. Ann Clin Biochem. 2011;48(Pt 6): 498-515.

45. Ginsberg HN, Fisher EA. The ever-expanding role of degradation in the regulation of apolipoprotein B metabolism. J Lipid Res. 2009;50(Suppl):S162-6.

46. Rutledge AC, Su Q, Adeli K. Apolipoprotein B100 biogenesis: a complex array of intracellular mechanisms regulating folding, stability, and lipoprotein assembly. Biochem Cell Biol. 2010;88(2):251-67.

47. Adeli K, Taghibiglou C, Van Iderstine SC, Lewis GF. Mechanisms of hepatic very low-density lipoprotein overproduction in insulin resistance. Trends Cardiovasc Med. 2001;11(5):170-6.

48. Qiu W, Zhang J, Dekker MJ, Wang H, Huang J, Brumell JH, et al. Hepatic autophagy mediates endoplasmic reticulum stress-induced degradation of misfoldedapolipoproteinB. Hepatology. 2011;53(5):1515-25.

49. Ohsaki Y, Cheng J, Fujita A, Tokumoto T, Fujimoto T. Cytoplasmic lipid droplets are sites of convergence of proteasomal and autophagic degradation of apolipoprotein B. Mol Biol Cell. 2006;17(6):2674-83.

50. Schröder M, Kaufman RJ. The mammalian unfolded protein response. Annu Rev Biochem. 2005;74:739-89.

51. Caviglia JM, Gayet C, Ota T, Hernandez-Ono A, Conlon DM, Jiang $\mathrm{H}$, et al. Different fatty acids inhibit apoB100 secretion by different pathways: unique roles for ER stress, ceramide, and autophagy. J Lipid Res. 2011;52(9):1636-51.

52. Pan M, Maitin V, Parathath S, Andreo U, Lin SX, St Germain C, et al. Presecretory oxidation, aggregation, and autophagic destruction of apoprotein-B: a pathway for late-stage quality control. Proc Natl Acad Sci U S A. 2008;105(15):5862-7. 
53. Muller C, Salvayre R, Nègre-Salvayre A, Vindis C. HDLs inhibit endoplasmic reticulum stress and autophagic response induced by oxidized LDLs. Cell Death Differ. 2011;18(5):817-28.

54. Martin S, Parton RG. Lipid droplets: a unified view of a dynamic organelle. Nat Rev Mol Cell Biol. 2006;7(5):373-8.

55. Hansson GK, Libby P. The immune response in atherosclerosis: a double-edged sword. Nat Rev Immunol. 2006;6(7):508-19.

56. Ouimet M. Autophagy in obesity and atherosclerosis: Interrelationships between cholesterol homeostasis, lipoprotein metabolism and autophagy in macrophages and other systems. Biochim Biophys Acta. 2013;1831(6):1124-33.

57. Tao R, Hoover HE, Honbo N, Kalinowski M, Alano CC, Karliner JS, et al. High-density lipoprotein determines adult mouse cardiomyocyte fate after hypoxia-reoxygenation through lipoprotein-associated sphingosine 1-phosphate. Am J Physiol Heart Circ Physiol. 2010;298(3):H1022-8.

58. Huang J, Lam GY, Brumell JH. Autophagy signaling through reactive oxygen species. Antioxid Redox Signal. 2011;14(11):2215-31.

59. Yorimitsu T, Nair U, Yang Z, Klionsky DJ. Endoplasmic reticulum stress triggers autophagy. J Biol Chem. 2006;281(40):30299-304.

60. Matsuzawa T, Kim BH, Shenoy AR, Kamitani S, Miyake M, Macmicking JD. IFN- $\gamma$ elicits macrophage autophagy via the p38 MAPK signaling pathway. J Immunol. 2012;189(2):813-8.

61. Karantza-Wadsworth V, Patel S, Kravchuk O, Chen G, Mathew R, Jin S, et al. Autophagy mitigates metabolic stress and genome damage in mammary tumorigenesis. Genes Dev. 2007;21(13):1621-35.

62. Razani B, Feng C, Coleman T, Emanuel R, Wen H, Hwang S, et al. Autophagy links inflammasomes to atherosclerotic progression. Cell Metab. 2012;15(4):534-44.

63. Saitoh T, Fujita N, Jang MH, Uematsu S, Yang BG, Satoh T, et al. Loss of the autophagy protein Atg16L1 enhances endotoxin-induced IL-1beta production. Nature. 2008;456(7219):264-8.

64. Levine B, Mizushima N, Virgin HW. Autophagy in immunity and inflammation. Nature. 2011;469(7330):323-35.

65. Liao X, Sluimer JC, Wang Y, Subramanian M, Brown K, Pattison JS, et al. Macrophage autophagy plays a protective role in advanced atherosclerosis. Cell Metab. 2012;15(4):545-53.

66. Zang L, He H, Ye Y, Liu W, Fan S, Tashiro SI, et al. Nitric oxide augments oridonin-induced efferocytosis by human histocytic lymphoma U937 cells via autophagy and the NF-kappaB-COX-2-IL1beta pathway. Free Radic Res. 2012;46(10):1207-19.

67. Fujitani Y, Ueno T, Watada H. Autophagy in health and disease. 4. The role of pancreatic beta-cell autophagy in health and diabetes. Am J Physiol Cell Physiol. 2010;299(1):C1-6.

68. Zick Y. Insulin resistance: a phosphorylation-based uncoupling of insulin signaling. Trends Cell Biol. 2001;11(11):437-41.
69. Posadas-Romero C, Mendoza-Pérez E, Juarez-Rojas JG, GómezSaviñón VG. Síndrome metabólico y diabetes. In: Martinez-Ríos MA, editor. Tratado de cardiología. México: Intersistemas; 2012. p. 343-57.

70. Ichimura Y, Kominami E, Tanaka K, Komatsu M. Selective turnover of p62/A170/SQSTM1 by autophagy. Autophagy. 2008;4(8): 1063-6.

71. Wu JJ, Quijano C, Chen E, Liu H, Cao L, Fergusson MM, et al. Mitochondrial dysfunction and oxidative stress mediate the physiological impairment induced by the disruption of autophagy. Aging (Albany NY). 2009;1(4):425-37.

72. Ebato C, Uchida T, Arakawa M, Komatsu M, Ueno T, Komiya K, et al. Autophagy is important in islet homeostasis and compensatory increase of beta cell mass in response to high-fat diet. Cell Metab. 2008;8(4):325-32.

73. Marsh BJ, Soden C, Alarcón C, Wicksteed BL, Yaekura K, Costin $\mathrm{AJ}$, et al. Regulated autophagy controls hormone content in secretory-deficient pancreatic endocrine beta-cells. Mol Endocrinol. 2007;21(9):2255-69.

74. Zhang Y, Zeng X, Jin S. Autophagy in adipose tissue biology. Pharmacol Res. 2012;66(6):505-12.

75. Mueller MA, Beutner F, Teupser D, Ceglarek U, Thiery J. Prevention of atherosclerosis by the mTOR inhibitor everolimus in LDLR-/ -mice despite severe hypercholesterolemia. Atherosclerosis. 2008;198(1):39-48

76. Pakala R, Stabile E, Jang GJ, Clavijo L, Waksman R. Rapamycin attenuates atherosclerotic plaque progression in apolipoprotein $\mathrm{E}$ knockout mice: inhibitory effect on monocyte chemotaxis. J Cardiovasc Pharmacol. 2005;46(4):481-6.

77. Basso MD, Nambi P, Adelman SJ. Effect of sirolimus on the cholesterol content of aortic arch in ApoE knockout mice. Transplant Proc. 2003;35(8):3136-8.

78. Morrisett JD, Abdel-Fattah G, Hoogeveen R, Mitchell E, Ballantyne $\mathrm{CM}$, Pownall HJ, et al. Effects of sirolimus on plasma lipids, lipoprotein levels, and fatty acid metabolism in renal transplant patients. J Lipid Res. 2002;43(8):1170-80.

79. Verheye S, Martinet W, Kockx MM, Knaapen MW, Salu K, Timmermans JP, et al. Selective clearance of macrophages in atherosclerotic plaques by autophagy. J Am Coll Cardiol. 2007;49(6):706-15.

80. Kasiske BL, de Mattos A, Flechner SM, Gallon L, Meier-Kriesche HU, Weir MR, et al. Mammalian target of rapamycin inhibitor dyslipidemia in kidney transplant recipients. Am J Transplant. 2008;8(7):1384-92.

81. Schrijvers DM, De Meyer GRY, Martinet W. Autophagy in atherosclerosis: a potential drug target for plaque stabilization. Arterioscler Thromb Vasc Biol. 2011;31(12):2787-91. 